\title{
Zwischen Verstehen und Verweisen (Post-)Migrationsgesellschaftliche Perspektiven auf die Vermittlung von Deutsch als Fremdsprache
}

\author{
Between Comprehension and Direction \\ The Concept of Post-Migration and the Teaching of German \\ as a Foreign Language
}

Constantin Wagner

\begin{abstract}
This paper attempts to reconstruct different paradigms, attitudes and experiences that underlie the understanding and action of non-professional teachers of German as a foreign language. The analysis shows that some of these teachers explain the problems of their students via a national-cultural affiliation, while other attempts to understand the students' challenges can also be observed. Participating observation of an academic congress on German as a foreign language makes clear that competing explanatory approaches are also present in specialist discourse. In this respect, the divergent categories of explanations observed in the field may appear to be based less on a teachers' lack of disciplinary socialisation than on different migration and diversity-related attitudes and views that originate from different social milieus.
\end{abstract}

\section{Keywords}

Post-Migration; German as a Foreign Language; Culturalism; Content Analysis; Participating Observation 


\section{Einleitung}

Der vorliegende Artikel stellt den Beitrag einer Person dar, die sich mit (migrationsbezogener) Heterogenität und sozialer Ungleichheit auseinandersetzt und vor allem zu diversitätsbezogenen Lern- und Bildungsprozessen arbeitet und nimmt insofern eine Outsider-Perspektive auf Sprachvermittlung ein. Weitestgehend ohne einen Hintergrund sprachwissenschaftlicher oder -didaktischer Expertise geht es darum, in der Auseinandersetzung mit empirischem Material (post-)migrationsgesellschaftliche, rassismuskritische Perspektiven auf den DaF-Kontext anzuwenden.

Hierfür werden nach einer Einführung zu der Bedeutung ethnisierend-kulturalsisierenden „Wissens“ und dessen Einfluss auf „Diagnose“ und „Behandlung“ im pädagogischen Kontext zunächst das primäre Datenmaterial sowie der methodische Zugriff hierauf vorgestellt; anschließend wird die Auswertung desselben präsentiert. Hierbei zeigen sich (u.a.) sowohl kulturalistische Erklärungsansätze als auch solche, die viel stärker auf den sozialen Kontext der Deutschlernenden abheben. Eine ethnografische Annäherung an den Fachdiskurs verdeutlicht, dass in demselben konkurrierenden Erklärungsansätze - und dabei sowohl aus (post-)migrationsgesellschaftlicher Perspektive problematische Kategorisierungen als auch progressive Ansätze - gleichzeitig vorhanden sind.

\section{Ethnisierend-kulturalisierendes „Wissen" und sein Einfluss auf "Diagnose" und „Behandlung" im pädagogischen Kontext}

Unterschiedliche Studien aus dem Kontext der Erziehungswissenschaft sowie der Wissenschaft Sozialer Arbeit haben herausgearbeitet, dass ethnisierend-kulturalisierende Vorstellungen von Adressat*innen einen Einfluss auf „Diagnose“ und „Behandlung“ im professionellen Handeln hat. So arbeitet Hahn (2011: 300) heraus, dass ethnische Differenzierungen zu einem erheblichen Maße dazu beitragen, dass „ein spezifisches Beziehungsgefüge zwischen den Gesprächspartnern konstituiert und über den Beratungsverlauf hinweg reproduziert wird“. In meiner Fallstudie zum Sozialamt (Wagner 2016, Wagner 2017) zeigt sich ebenfalls, dass die wahrgenommene ethnische Zugehörigkeit einen Einfluss auf die Beziehung, Beurteilung und Beratung in professionellen pädagogischen Beziehungen haben kann. Besonders deutlich wird dies in einem Interview mit der Sozialarbeiterin Birgit ${ }^{1}$ :

„[I]ch denke, wir haben eine große... also sagen wir jetzt, die (...) Politik eine große Mitschuld daran trägt, weil wir ihnen von Anfang... (...) eigentlich alles auf dem Tablett servieren. Und wie sollen die das verstehen, dass das nicht anders ist? Da tun wir eigentlich... Wie sollen wir ihnen das klarmachen, dass bei uns die 100er Noten nicht auf den Bäumen wachsen, dass wir auch arbeiten für unser Geld. Und dann nachher (.) die Sozialhilfe andere Rahmenbedingungen hat, als zum Beispiel der Bund, der natürlich die Flüchtlinge in den ersten 5 Jahren unter- 
stützt, vielmehr Geld fließen lässt. Aber das ist nicht bei allen so. Es gibt einfach solche, die... es gibt ja auch sagen wir jetzt (.) aus Afrika zum Beispiel, in Afrika, sei es in Ghana, Nigeria, Uganda, (.) Burundi, so diese Regionen, gibt es unterschiedliche Klassen. Nicht nur Stämme, sondern auch Klassen. Dann gibt es Klassen, (.) die arbeiten und es gibt Klassen, die arbeiten lassen. Und die, die (...) kommen, sind in der Regel die, die arbeiten lassen. Jetzt bringen Sie einmal so jemanden zu einem Job. [lacht]. Das kennt der doch gar nicht. Der hat das mit der Muttermilch schon aufgenommen, dass man nicht arbeitet, sondern arbeiten lässt. Das ist eine andere Kultur, die sich einfach nicht bewusst ist. Weil der, der arbeiten muss aus dieser Klasse, der kann sich das gar nicht leisten, zu flüchten. Ein anderer kommt gar nicht auf die Idee, dass er vielleicht irgendwo... oder hat nicht einmal den Zugang, dass es irgendwo anders sein könnte.“

Die Sozialarbeiterin hat (zum Teil sehr spezielle) ethno-kulturalistische Erklärungsansätze, mit denen sie ihre Klient*innen und deren Verhalten einordnet, erklärt und auf dieser Grundlage beurteilt (und behandelt). Die Tatsache, dass Ghana und Burundi mehr als 5.000 Kilometer voneinander entfernt sind und Ghana näher an Spanien liegt als an Burundi, hält sie nicht davon ab, in Bezug auf die beiden afrikanischen Länder von „diesen Regionen“ zu sprechen. Sie scheint hier von rassifizierenden Vorstellungen von einem „Schwarzafrika“ geprägt zu sein. Von einer ähnlichen „ethnischen“ Kategorisierung lässt sich dort ausgehen, wo sie von einem „Schlüsselerlebnis“ berichtet, in dem „ein albanischer Staatsbürger oder ein türkischer“ der Protagonist ist. Sie beschreibt, dass dieser die Terminkärtchen, die er in der Arztpraxis am Ende des Besuchs erhalten hat, als Krankschreibung interpretiert. Für Birgit sei dies ein Schlüsselerlebnis gewesen insofern, als dass sie hier gemerkt habe, dass "diese Leute“ hierzulande keine Orientierungskompetenz haben. Sie schließt dabei von persönlichen Erfahrungen mit einzelnen Personen auf eine (so nicht vorhandene) Gruppe; sie aktiviert bei diesem Schluss gesellschaftlich-kulturell tradiertes, orientalistisches Wissen über „Kulturkreise“ („ein albanischer Staatsbürger oder ein türkischer“). Erst dies erlaubt ihr, die Erfahrungen mit einzelnen Klient*innen auf bestimmte andere Klient*innen zu übertragen. Ferner verknüpfen sich ihre kulturalistischen Erklärungen mit nationalistischen Diskursen um Nützlichkeit und Leistungsfähigkeit, die politisch sehr präsent sind. Während Birgit ein Extremfall hinsichtlich der offenen Artikulation kulturrassistischer Vorstellungen ist, zeigt sich eine - in aller Regel viel subtilere, weniger abwertende und den Protagonist*innen selbst weniger bewusste - Verknüpfung von Ethnizität und Beurteilung von Fällen bei sehr vielen Sozialarbeiter*innen.

Die (vermutete) ethnische Zugehörigkeit beeinflusst - je nach Sozialarbeiter*in unterschiedlich stark und in unterschiedlicher Weise - die Beurteilung der Legitimität von Transferleistungen sowie die Einschätzung der Leistungsfähigkeit der Klient*innen und deren Chancen auf gesellschaftlich-beruflichen Erfolg. Ferner ist fraglich, inwiefern die Sozialarbeitenden ein grundlegendes Verständnis für die Lebenssituation der jeweiligen Klient*in entwickeln können. (Geteilte) „Ethnizität“ wirkt sich auch auf das Gefühl von Nähe und Distanz und somit auf die Möglichkeit aus, eine professionelle Unterstützungsbeziehung aufzubauen. Dies lässt sich an der Episode „Gebückte Haltung“ 
gut nachvollziehen: Birgit erzählt mir, dass ich darauf achten solle, wenn Klient*innen aus der Balkanregion ins Sprechzimmer kommen würden, wie sehr sie eine gebückte, bettelnde Körperhaltung einnehmen würden. Man könne daran sehen, dass sie nicht gewillt seien, selbst Verantwortung für ihre Situation zu übernehmen und stattdessen eine fordernde Haltung gegenüber dem/der Sozialarbeiter*in einnehmen würden. Monika, eine andere Sozialarbeiterin, die selbst mit ihrer Familie in der Balkanregion aufgewachsen und später in die Schweiz migriert ist, spricht von dem gleichen Personenkreis und nimmt eine ähnliche Körperhaltung wahr; liest bzw. deutet diese aber gänzlich anders. Sie sagt zu mir, diese Haltung zeige, dass hier häufig Leute ins Zimmer kommen würden, die über Jahre und Jahrzehnte schwere körperliche Arbeit auf dem Bau oder in der Industrie verrichtet hätten - und trotzdem auf soziale Unterstützung angewiesen seien, weil man sie so schlecht entlohnt habe. Man könne ihrem Körper ihre soziale Position innerhalb der schweizerischen Gesellschaft förmlich ansehen. Es mag auf Grundlage dieser unterschiedlichen Einschätzungen kaum verwundern, dass Monika und Birgit die entsprechenden Klient*innen sehr unterschiedlich „behandeln“. Diese Kontrastierung zweier unterschiedlicher Fallverständnisse zeigt, dass die „weiße“, d.h. dominanzgesellschaftliche Sicht Birgits perspektiviert werden kann und es alternative Sichtweisen auf denselben Sachverhalt gibt, die möglicherweise eine stärkere Erklärungskraft haben. Interessant ist nun, dass Monika ihre Erklärung, die die (spezifische) soziale Situation der Klient*innen viel stärker in Betracht zieht als die kulturalisierend-ethnisierende Sichtweise Birgits, etwa in Teambesprechungen und bei anderen Gelegenheiten, bei denen kollektive Sichtweisen auf „Fälle“ produziert werden, nicht anbringt. Sie ist marginalisiert, wäre selbst als „Betroffene“ angreifbar und ist so nicht sprachfähig: Sie ist die einzige Sozialarbeiterin of Colour im Team.

Die Aktivierung von ethnisierend-kulturalisierendem Wissen und ein gleichzeitiges Unwissen im Hinblick auf die Bedeutung einer rassifizierten gesellschaftlichen Struktur hat ferner eine „produktive“ Funktion im beruflichen Kontext und mag auch deswegen sehr hartnäckig sein: Sie leistet eine Komplexitätsreduktion und eine Vereindeutigung der Problemlage der/des jeweilige/n Klient*in. Nicht die (träge!) gesellschaftliche Struktur ist die zu verändernde Größe, sondern der/die Klient*in. Individuelle ethnisierend-kulturalisierende „Wissensbestände“ werden in Fallbesprechungen, Tür-und-Angel-Gesprächen sowie im Pausenraum zu kollektiv geteiltem professionellen Wissen, etwa über die Sammlung „kurioser Fälle“. Das hier als solches bezeichnete „professionelle Wissen“ interagiert sehr stark mit dem an das im Herkunftsmilieu einer rassifizierten Gesellschaft erworbene Alltagswissen der dominierenden Akteur*innen und ist kein Wissen, das kritisch-reflektierend überprüft wurde; es ist trotzdem „professionelles Wissen“, weil es eine berufliche Funktion erfüllt: Die ethno-kulturelle Zugehörigkeit nimmt Einfluss auf die Fallführung der Sozialarbeitenden (Was ist „das Problem“ des jeweiligen Falls?; In welche Fälle lohnt sich ein Investment an Budget und Zeit?). Eine Einordnung der Fälle über eine national-ethno-religiös-kulturelle Kategorisierung ist dabei, wie gesagt, durchaus auch bei Sozialarbeitenden beobachtbar, die (anders als Birgit) ihren Klient*innen of Color grundsätzlich mit Sympathie begegnen. Dass ethnisierend-kulturalisierendes Wissen in der Institution dominant werden kann, verweist dabei direkt auf 
die Struktur der Mitarbeitenden und ihre jeweiligen Erfahrungshintergründe.

Im Folgenden soll diskutiert werden, inwiefern diese Perspektive auf Fallverstehen und professionelles Handeln auf den Unterricht nicht-professioneller DaF-Lehrer*innen anzuwenden ist.

\section{Datenerhebung und methodischer Zugriff}

Die für den vorliegenden Artikel ausgewerteten Primärdaten stammen aus einem Projekt, in dem Studierende DaF an Geflüchtete unterrichtet haben. Um das Fallverstehen beziehungsweise die Problemkonstruktion der nicht-professionellen Lehrkräfte nachzuvollziehen, wurden sie gefragt, was ihren Schüler*innen Schwierigkeiten beim Deutschlernen bereitet. Als Vorbereitung einer Supervisionssitzung sollten sie hierzu Stichpunkte oder einen Fließtext aufschreiben.

Die von den Unterrichtenden schriftlich fixierten Begründungsmuster wurden hier zunächst nach dem Verfahren der qualitativen Inhaltsanalyse nach Mayring (2000a, 2000b) induktiv kategorisiert: Im ersten Schritt erfolgte hierfür eine sprachlich nah am Originaltext bleibende Paraphrasierung der Texte und anschließende (verkürzte) Problembenennung zum Zwecke der Reduktion des Materials (inklusive Benennung der vermuteten Problemursache, sofern vorhanden). Dieser erste Schritt der Datenaufbereitung generierte eine Übersicht, die verdeutlicht welche Themen von den ehrenamtlich Lehrenden benannt wurden.

In einem zweiten Schritt mussten die unterschiedlichen Problembenennungen kategorisiert werden. Die im ersten Schritt vorgenommenen Verdichtungen konnten die Grundlage für die Entwicklung der Kategorien sein, mit deren Hilfe sich Verbindungen zwischen unterschiedlichen Textstellen und Einzelfällen herstellen lassen (Kühn - Witzel, 2000); die Problembenennungen konnten so in vier unterschiedliche Kategorien geclustert werden.

In einem dritten Schritt konnte im Anschluss an diese inhaltsanalytische Kategorisierung des Materials eine Falldarstellung erstellt werden. Diese Falldarstellungen stehen jeweils prototypisch für die einzelnen Kategorien von Problemwahrnehmungen. In den Falldarstellungen lassen sich Originaltextstellen, Paraphrasierungen und analytische Aussagen verdichten und verbinden (Strauss - Corbin 1990).

Ergänzend zu dieser Erhebung und auf Grundlage der Ergebnisse derselben (nämlich, dass kategorial sehr unterschiedliche Erklärungsmuster zu beobachten sind) wurde der Fachdiskurs auf einem Arbeitstreffen der Sektion einer akademischen Fachgesellschaft, die sich mit DaF für Geflüchtete beschäftigt, ethnografisch im Hinblick auf die Frage nach der Präsenz divergenter Verständnisse von den Herausforderungen des Deutschlernens im Kontext von Fluchtmigration beobachtet. Im Zentrum dieser ethnografischen Datenerhebung steht dabei das Feldgeschehen und die dabei zu beobachtenden Handlungspraktiken - und nicht die Einzelpersonen, -projekte und ihre Intentionen (Schulz 2014). Abstrahiert von den konkreten Akteur*innen und Forschungsprojekten sollte der Feldaufenthalt zeigen, was sag- und denkbar ist. Zu diesem Zwecke wurden 
Vorträge und Diskussionen protokolliert und im Anschluss daraufhin befragt, inwiefern die im ersten Erhebungsschritt herausgearbeiteten Kategorien hier ebenso relevant sind. In ethnografischen Verfahren stehen Datenerhebung und -auswertung in einem sich aufeinander beziehenden Verhältnis und sind weniger klar analytisch zu trennen (Breidenstein 2006; Breidenstein 2010); der ethnografische Zugriff privilegiert einen offenen, explorierenden Zugang zum Feld, gleichzeitig können ethnografische Untersuchungen die gebotene Offenheit mit einem fokussierten Blick verbinden (Knoblauch 2001). Aus pragmatischen Gründen bleibt der ethnografische Zugriff auf den Fachdiskurs exemplarisch; die Ergebnisse sollen die Erkenntnisse der Primäruntersuchung lediglich kontextualisieren.

\section{Fallverstehen / Problemkonstruktionen von ehrenamtlich DaF-Lehrkräften}

Die Primärdaten stammen aus einem im Jahr 2015 begonnenen Projekt einer deutschen Universität, in dem Studierende ehrenamtlich Geflüchtete unterrichten, „die Deutsch lernen möchten, um sich hier in Deutschland besser integrieren zu können“2. Der Unterricht findet vor allem in Erstaufnahmeeinrichtungen, aber auch an anderen Orten, statt. Zur Teilnahme an dem Programm mussten sich die Studierenden bewerben, erhielten anschließend eine zweitägige Schulung und unterrichteten dann im Tandem zwei Mal die Woche. Begleitet wurden sie dabei durch eine alle zwei bis drei Wochen stattfindende Supervisionssitzung. Zur Vorbereitung auf eine solche Supervisionssitzung gab ich der Gruppe von zwanzig ehrenamtlich Lehrenden folgende Aufgabe: „Was sind die zentralen Herausforderungen der Schüler*innen beim Deutsch-Lernen und woher kommen diese Herausforderungen?" Die (unterschiedlichen) wahrgenommenen Herausforderungen wurden in der Supervisionssitzung besprochen.

\subsection{Wahrgenommene Problematiken}

Benannt wurden von den ehrenamtlich Lehrenden folgende Herausforderungen (sprachlich nah am Original bleibende, paraphrasierende Darstellung; die vermutete Problemursache, so benannt, in Klammern hinter den Problembenennungen):

- Rechtschreibung (unterschiedliche Herkunfts-Phonetik);

- Groß-/Kleinschreibung (fehlende Konzentration);

- Schreibfaulheit;

- Flüchtigkeitsfehler;

- korrekte, deutliche Aussprache;

- Textverständnis;

- Schwächen / Lücken zugeben;

2 Aus Gründen der Anonymisierung erfolgt hier keine genaue Zitation. 
- mangelnde Alltagsanwendung (Isolation in Wohnanlage / kein Austausch mit Muttersprachlern);

- Motivation, regelmäßig zu kommen (Unsicherheit, ob sich Investment lohnt / weiter Anfahrtsweg / allg. Motivationslosigkeit);

- Kontinuität fehlt (keine generelle Anwesenheitspflicht);

- Frustration (Abwertung des Qualifikationsniveaus aus Heimat);

- Hunger wegen Fasten (Ramadhan / zu geringer Pragmatismus);

- Heterogenität in der Lerngruppe;

- Nicht-hier-sein-wollen und gleichzeitig Heimat nicht freiwillig verlassen, um Aufenthaltstitel kämpfen;

- Nichts machen können (Keine Arbeits- oder Studiengenehmigung, Sprachdefizite);

- sich nicht erwünscht fühlen (Medien, Übergriffe im Alltag);

- Unsicherheit, ob man sich an neue Umgebung gewöhnen kann (unklar, ob man bleibt);

- Keine deutschen Freunde;

- (für Kinder) Erwachsenenaufgaben übernehmen müssen, gleichzeitig für Eltern: Können ihren Erwachsenenaufgaben nicht nachkommen (Kinder können Sprache besser als Eltern)

\subsection{Kategorien von Problemwahrnehmungen}

Diese, teilweise sehr unterschiedlichen, wahrgenommenen Problematiken lassen sich in vier unterschiedliche Kategorien clustern:

Eine erste Kategorie von wahrgenommenen Problemen bezieht sich auf die konkrete jeweilige Lerngruppe bzw. das Setting (und somit nicht auf die Lernenden als solche). Beispiele hierfür sind die Nennungen „Heterogenität in der Lerngruppe“ oder „Kontinuität fehlt (keine generelle Anwesenheitspflicht)“.

Eine zweite Kategorie der vermuteten Herausforderungen der Schüler*innen bezieht sich auf die soziale Situation derselben. Beispiele hierfür sind „Mangelnde Alltagsanwendung (Isolation in Wohnanlage / Kein Austausch mit Muttersprachlern)“, „Frustration (Abwertung des Qualifikationsniveaus)“ oder „Motivation, regelmäßig zu kommen (Unsicherheit, ob sich Investment lohnt / weiter Anfahrtsweg / allg. Motivationslosigkeit)“.

Eine dritte Kategorie bildet die kulturelle Prägung bzw. die (vermutete) kulturelle Zugehörigkeit der Schüler*innen als wahrgenommene Problematik für das Deutschlernen. Beispiele hierfür sind „Schwächen / Lücken zugeben“, „Schreibfaulheit“ oder „Hunger wegen Fasten (Ramadhan / zu geringer Pragmatismus)“.

Eine vierte Kategorie bezieht sich ausschließlich auf den Fremdsprachenerwerb als solchen und nimmt eine technische Perspektive auf den Spracherwerb ein, sie kommt ohne Erklärung außerhalb des Sprachenlernens aus. Beispiele hierfür sind „Flüchtigkeitsfehler“ oder „Groß-/Kleinschreibung (fehlende Konzentration)“.

Die inhaltsanalytische Auswertung der wahrgenommenen Herausforderungen verdeutlicht zunächst, dass bei den ehrenamtlich Lehrenden unterschiedliche Kategorien 
und Ebenen von wahrgenommenen Problematiken vorhanden sind. Zum (geringeren) Teil werden dabei von Lehrenden Erklärungen unterschiedlicher Kategorien genannt; zumeist nehmen einzelne Lehrkräfte Herausforderungen jeweils einer Kategorie wahr. Im Folgenden soll jeweils ein Fall, der prototypisch für die entsprechende Kategorie steht, interpretierend dargestellt werden.

\subsection{Falldarstellungen}

Der Text von Britta steht stellvertretend für die erste Kategorie, die sich im Hinblick auf Herausforderungen für die Schüler*innen auf die jeweilige Lerngruppe bzw. das Setting bezieht. Dabei bleibt oft unklar, ob die genannten Punkte tatsächlich Herausforderungen für die Lernenden oder eher für die Lehrenden sind:

„Keine Verpflichtung jeden Tag zu kommen, weshalb teilweise die Kontinuität fehlt $\rightarrow$ einige
sind immer da, andere nur ab und zu. Innerhalb der Gruppe sind oft große sprachliche Dif-
ferenzen zu finden $\rightarrow$ während die einen noch immer an der ersten Aufgabe sitzen, haben
andere bereits das ganze Arbeitsblatt gearbeitet $\rightarrow$ hier mehr Angebote schaffen, sodass sich
die besseren Schüler*innen nicht anfangen zu langweilen. Aufgrund von drei verschiedenen
Sprachniveau-Gruppen die gleichzeitig in einem Raum unterrichtet werden kann es teilweise
ziemlich laut und auch durcheinander werden. (...)“

Dass einige Lernende immer da sind, während andere nur ab und zu kommen scheint eher für die Lehrenden ein Problem zu sein; auch gibt der Text bereits Hinweise auf einen möglichen didaktischen Umgang mit der Problematik. Britta nimmt es wohl für sich selbst, aber auch auf ihre Schüler*innen, als schwierig wahr, in dem gegebenen Setting einen Unterricht durchzuführen, von dem alle profitieren und in dem alle gut lernen können. Die weiteren Punkte, die Britta benennt, könnten auch in die vierte Kategorie („technische Perspektive auf den Spracherwerb“) gruppiert werden:

„(...) Buchstaben werden teilweise noch falschherum geschrieben $\rightarrow$ mehr Schreibübungen würden hierbei helfen, aber auch eine erneute und vertiefende Alphabetisierung (...) Mehr Abwechslung schaffen: auch wenn z.B. bei den Buchstaben oder Artikeln noch Defizite herrschen, dennoch auch andere Angebote schaffen: Verben einführen, erste kurze Sätze bilden o.ä., um die Schüler*innen weiter zu motivieren."

Von diesem Erklärungsansatz unterscheidet sich der Text von Yeliz kategorial. Als Herausforderungen der Schüler*innen benennt sie primär deren soziale Situation.

„Herausforderungen. Sprachlich: Alltägliche Anwendung \& Umsetzung des gelernten Stoffes. Insbesondere wenn es über Standart-Situationen (Bus, Einkaufen etc.) hinaus geht. Gespräche führen, diskutieren, Meinungsaustausch. Warum? Oft sind die Kreise in denen sich die SchülerInnen bewegen überwiegend nicht deutschsprachig. Im Unterricht bleibt es immer auf einer 
Exemplarischen Ebene. Allgemein: Langfristige Perspektiven, Pläne und Ziele nicht verlieren / wachhalten / entwickeln.. Warum? Unsicherheit was den Aufenthaltsstatus angeht, Schwierigkeiten eine Wohnung / Job zu finden...“

Yeliz hinterfragt hier den dominanzgesellschaftlichen ${ }^{3}$ Diskurs von Sprache als „Schlüssel“ oder hinreichende Bedingungen für „Integration“. Deutsch erscheint hier nicht als Ansammlung von grammatischen und phonologischen Fertigkeiten oder technisch zu vermittelnden lexikalischen Kompetenzen, sondern als sich durch soziale Erfahrungen und konkrete Interaktionen ausprägend. Yeliz geht es um die Vermittlung von Sprachfähigkeit und Verständigung; sie verortet sich selbst wesentlich weniger als Expertin in der Sprachvermittlung als Lehrende, deren Sichtweise in der ersten oder vierten Kategorie geclustert wurden. Ihr Ansatz könnte als lebensweltorientiert beschrieben werden, während die Sichtweise der ersten (sowie der vierten) Kategorie eine Fehlerorientierung aufweisen.

Der Text, der die Überschrift „Herausforderung für ,meine‘ Flüchtlinge“ trägt und der von Ruth geschrieben wurde, ist ein emotionaler Text, der exemplarisch für die Kategorie von Problemwahrnehmungen steht, die die kulturelle Prägung bzw. die (vermutete) kulturelle Zugehörigkeit der Schüler*innen für zentral halten. Auffällig ist, dass Ruth unübliche Formulierungen benutzt, die sich möglicherweise an Aussagen der Schüler*innen orientieren:

„(...) Auch die anderen Männer kommen regelmäßig (...). Sie sind alle willige Schüler und Schülerinnen. (...) Noch leben sie alle in einem ,Zwischenland‘. Sie haben ihre Heimat hinter sich gelassen. Und auch wenn sie dem Terror entkommen sind, schmerzt sie die Sehnsucht nach den Bergen Afghanistans oder den Gerüchen und Geräuschen in den Straßen Syriens. (...) Ihre Perspektive ist ungewiss. Dürfen sie bleiben? Sich ein neues Leben aufbauen in diesem so fremden Land? Wie soll das gehen ohne Sprache? Diese schwere Sprache, die der ihren so fremd ist. Zunge, Lippen und Ohr müssen den Unterschied zwischen u und ü erkennen und nachvollziehen."

Der Text von Ruth ist eine Für-Sprache für die von ihr unterrichteten Schüler*innen (und dabei möglicherweise zugleich eine Einverleibung derselben). ${ }^{4}$ Hanna zeigt sich durchaus kritisch gegenüber dominanten Wahrnehmungen, ihre Aussagen sind aber ambivalent: Dies kommt auch, aber nicht nur in der Überschrift ihres Textes („,meine“ Flüchtlinge“) zum Ausdruck. Der paternalistische Zugang scheint ebenso in der Formulierung „willig“ durch. Interessant ist auch die folgende Passage ihres Textes:

„J. macht den Haushalt, aber das ist er nicht gewohnt und mag es auch nicht. Aber er tut es. Weil es irgendjemand machen muss und weil seine Frau es nicht kann. Er und die anderen

3 Siehe zu dem Begriff Rommelspacher (2006).

4 Dazu passt, dass sich Ruth mit der Teilnahme an dem Projekt sozial engagieren will; gleichzeitig sieht sie darin eine Qualifikation für das Förderschullehramt (ihr Studiengang), dort habe sie auch „mit Migrationskindern zu tun“. Vor allem freue sie sich aber auf das Helfen. 
Männer müssen in ihren Rollenbildern von jetzt auf gleich Schritte tun, für die wir in Deutschland einige Jahrzehnte gebraucht haben (...).“

Die Sequenz zeigt, dass Ruth ihre Schüler*innen als Angehörige bzw. Merkmalsträger*innen einer Kultur versteht und dabei einer modernisierungstheoretischen Vorstellung folgt. Bleibt man in diesem Bild, sieht sich Ruth als eine Art „Entwicklungshelferin“, die ihre Schüler*innen im Modernisierungsprozess begleitet und sie phasenweise „an die Hand nimmt“. Ruth sagt bei der Diskussion der Texte, sie sehe sich nicht primär als Lehrerin; ihr sei die persönliche Beziehung sehr wichtig. ${ }^{5}$

Der Text von Jasmin ist hingegen ein Beispiel für die Kategorie von Problemwahrnehmungen, die ohne Erklärung außerhalb des Sprachenlernens auskommt. Die Perspektive bezieht sich ausschließlich auf den Fremdsprachenerwerb als solchen und nimmt eine technische Perspektive ein; relativ häufig, aber nicht immer, werden solche Erklärungen mit solchen aus der ersten Kategorie („Setting/Lerngruppe“) kombiniert. Jasmin schreibt:

„dennoch gibt es fundamentale Rechtschreibfehler/-problematiken wie Groß- und Kleinschreibung, Satzstellung und Fehlern die mit den unterschiedlichen Phonetiken der Sprachen zu tun haben, wie bspw. ,seher' statt ,sehr'; speziell bei letzterem weiß ich nicht wie man diese idealerweise trainieren kann; die phonetische Barriere sehe ich nicht nur zwischen Deutsch und der jeweiligen Fremdsprache sondern auch dass es große Unterschiede zwischen den fremden Sprachen mit individuellen Schwierigkeiten gibt und ich habe mir dazu Gedanken gemacht ob man diese Heterogenität optimieren kann oder ob es da Tipps gibt“

Die Herausforderung der Schüler*innen scheinen sich aus dieser Perspektive mit guten Lehrstrategien bearbeiten zu lassen. Heterogenität wird hier zunächst als Herausforderung verstanden, die aber im Unterricht „optimiert“ werden kann. Durch den funktional-sprachdidaktischen Zugriff rücken Fragen nach dem Zusammenhang von Sprache und gesellschaftlichen (Macht-)Verhältnissen aus dem Blick und solche zur didaktischen Gestaltung des Unterrichts in den Blick. Die Idee eines funktionalen Sprachenlernens privilegiert, überspitzt gesagt, den Zwang (zur Teilnahme), während sich die lebensweltliche Orientierung auf die Motivation der Teilnehmenden bezieht. Vergleicht man den Ansatz, der hier exemplarisch von Jasmin vertreten wird, mit dem, der die (vermutete) kulturelle Zugehörigkeit relevant setzt, fällt auf, dass hier weniger stark ethnisiert bzw. kulturalisiert wird. Gleichzeitig ist dieses Verständnis von Sprachenlernen nicht davor gefeit, von einer kulturalisierenden Denkweise unterfüttert zu werden, die sich auch im Text von Jasmin zeigt, wenn einer „phonetischen Barriere“ gesprochen wird und alle Sprachen außer Deutsch als „fremd“ markiert werden; fremd sind sie schließlich zunächst nur aus Sicht der Lehrkraft. Das eher technische Verständnis bzw. die rein sprachsystematisch/-didaktische Ebene, mit samt ihrer Fehler- bzw. Defizitorientierung, scheinen ferner wenig geeignet, die Bedingungen, unter denen gelernt wird, systema- 
tisch in den Blick nehmen zu können. Eine lebensweltorientierte Beschreibung ermöglicht Empathie und Gesellschaftskritik, ist aber ambivalent, weil sie - auch aufgrund von Viktimisierungstendenzen - „kippen“ kann.

Die unterschiedlichen Wahrnehmungen zeigen sich auch in der Diskussion der Texte im Rahmen der Supervisionssitzung. Als ich frage, warum die Schüler*innen denn „schreibfaul" seien, antwortet Andre, dass sie mehrheitlich aus oralen Kultur stammen würden und ihnen das Schreiben aus diesem Grunde nicht sehr geläufig sei und bezieht sich damit auf die (vermutete) kulturelle Zugehörigkeit der Schüler*innen. Meryem gibt darauf hin zu bedenken, dass sie glaube, dass die Schüler*innen sich scheuen zu schreiben, weil sie denken, dass sie nicht gut darin seien und sie so abgewertet werden könnten und bezieht sich damit zumindest implizit auf deren soziale Situation. Ruth nimmt danach Stellung und schließt ihrerseits an den kulturellen Frame an, wenn sie sagt, vielleicht sei den Schüler*innen das Schreiben auf Deutsch einfach zu fremd.

In Analogie zu der oben geschilderten Episode „Gebückte Haltung“ erscheint es dabei nicht zufällig, welche ehrenamtlich Lehrenden welche Problemwahrnehmungen und -erklärungen bevorzugen. Für Yeliz ist Deutsch selbst Zweitsprache, für Meryem ebenso. Für Ruth, Jasmin, Britta und Andre gilt dies nicht. ${ }^{6}$ Die Präsenz sehr unterschiedlicher, sich teilweise widersprechender Erklärungsansätze bzw. Problemwahrnehmungen provoziert vor diesem Hintergrund die Frage, inwiefern sie in einer Nichtsozialisation bzw. mangelnden Sozialisation in der Disziplin DaF begründet ist oder inwiefern sie für unterschiedliche, grundlegende migrations- und differenzbezogene Einstellungen und Anschauungen stehen, die in unterschiedlichen gesellschaftlichen Milieus beheimatet sind.

\section{Exkurs: Ethnografische Annäherungen an einen Fachdiskurs}

Aus diesem Grunde sollte der Fachdiskurs auf einem Arbeitstreffen der Sektion einer akademischen Fachgesellschaft, die sich mit DaF für Geflüchtete beschäftigt, ethnografisch beobachtet werden. Vor allem zum Zwecke der Anonymisierung werden von den konkreten Akteur*innen und Forschungsprojekten abstrahierend die Ergebnisse direkt verdichtend dargestellt. ${ }^{7}$ Der Feldaufenthalt sollte primär zeigen, was sag- und denkbar ist. $\mathrm{Zu}$ diesem Zwecke wurden Vorträge und Diskussionen protokolliert und im Anschluss daraufhin befragt, inwiefern die im ersten Erhebungsschritt herausgearbeiteten kategorial unterschiedlichen Ansätze hier ebenso relevant sind.

Tatsächlich zeigt sich, dass es in der disziplinären Diskussion Ansätze gibt, welche über eine andere Prämisse verfügen als der von den ehrenamtlich Lehrenden durchgeführte Unterricht und welche die Problemwahrnehmungen der Lehrenden vor dem Hintergrund eines grundlegend anderen Umgangs mit Sprache(n) transzendieren. Allerdings scheinen die unterschiedlichen Kongressbeiträge auch eine „Gleichzeitigkeit

6 Diese Beobachtung soll nicht nahelegen, dass ein Automatismus zwischen (sprachlicher) Herkunft und (Nicht-)Kulturalisierung bestehen würde.

7 Aus Gründen der Anonymisierung werden auch weder Ort noch Zeitpunkt des Arbeitstreffens genannt; auch Projekt- und Vortragstitel werden nicht zitiert. 
des Ungleichzeitigen“ zu verdeutlichen, denn parallel zu den Ansätzen, die auf der Grundlage eines neuen Paradigmas zu stehen scheinen, lässt sich ebenso eine nationalisierend-ethnisierend-kulturalisierende sowie eine technisch-didaktisierende Sichtweise auf den Unterricht und geflüchtete Deutschlernende beobachten, die sich nicht grundlegend von den Einschätzungen der ehrenamtlich Lehrenden unterscheiden.

Einerseits lässt sich eine Abkehr vom Paradigma der Einsprachigkeit, auch im Kontext Schule, beobachten: In den entsprechenden Projekten, wird Mehrsprachigkeit als Ressource für den Unterricht gedacht, sämtliche sprachliche Ressourcen sollen für fachliches Lernen und Verstehen nutzbar gemacht werden. Mehrsprachigkeit wird ferner auch auf Seiten der Lehrkräfte als Ressource gedacht; die konzeptionelle Aktivierung von L1 (und nicht: einer „Fremdsprache“) als Unterrichtssprache wird positiv bewertet, weil Verstehensprozesse durch Übersetzung angeregt werden können. Darüber hinaus wird die Aneignung metasprachlichen Wissens wertgeschätzt; Sprachenwechsel wird als Aktivierung von Wissensprozessen verstanden. Die mononlinguale Lehrkraft wird vor diesem Hintergrund eher zum Problem, weil sie schlechter mehrsprachige Ressourcen aktivieren kann, als zum unhinterfragten Normal- bzw. Prototypen einer Lehrkraft. Auch einige Projekte im außerschulischen Kontext betrachten die Erstsprache sowie die Bilingualität von Lehrkräften als Ressource.

Andererseits ist eine nationalisierend-kulturalisierende Sichtweise auf Deutschlernende auch auf dem Kongress präsent, etwa wenn in einem Vortrag konstatiert wird, Lernende aus Syrien seien in der Regel gut, aus Afghanistan okay, aus Somalia, Eritrea und Äthiopien schlecht. Die Ursachen für diese interpersonellen Unterschiede werden dabei über die Bildungssysteme in den Herkunftsländern erklärt; in Äthiopien sei „erst seit zwanzig Jahren Bildung entwickelt“. Auch wenn aus dem Plenum gefragt wird, ob nicht auch Einflussfaktoren in Deutschland in die Analyse einbezogen werden sollten, korrespondiert diese Problemwahrnehmung mit den kulturalisierenden Einschätzungen der ehrenamtlich Lehrenden sowie mit kulturalisierenden Wahrnehmungen und Diagnosen, die für den Bereich der Sozialen Arbeit festgestellt wurden. Auf dieser Linie bewegt sich auch eine Untersuchung, die die Wahrnehmung von Geflüchteten hinsichtlich ihrer studienvorbereitenden Deutschkurse erheben soll, deren subjektive Wahrnehmungen dann aber über nationale Kategorien erklärt. Ferner gibt es Projekte, die die Vermittlung von Deutsch als Einführung in eine Leitkultur verstehen und die eine „Passung“ von Geflüchteten in eine (scheinbar) bereits gegebene Gesellschafts(-ordnung) zum Ziel haben. Zielgruppe sind dementsprechend „Praktikanten, die aus anderen Ländern, mit anderen Kulturen kommen und nicht gut Deutsch sprechen“ und denen Sprachkenntnisse und gewünschte Verhaltensweisen bestimmter Industriezweige vermittelt werden sollen, in denen sie benötigt werden.

Die Unterschiedlichkeit der Kongressbeiträge verdeutlicht, dass die präsentierten Projekte und Ansätze sehr unterschiedliche Prämissen und Ziele verfolgen: Während einige versuchen, einen der Realität einer postmigrantischen Gesellschaft angemessenen Sprachunterricht zu modellieren, sind andere klar im Paradigma einer (Assimilations-) Integrationsvorstellung verhaftet. Letztere spanen sich - in gewisser Weise analog zu den Problemwahrnehmungen der ehrenamtlich Lehrenden - zwischen technisch-didaktisie- 
renden „Werkzeugkästen“ bis hin zu Versuchen, Menschen für einen bestimmten Gesellschaftsbereich passend zu machen. Obwohl hier offensichtlich gänzlich unterschiedliche Vorstellungen von Sprache(n) (und von Gesellschaft) zugrunde liegen, gibt es keine explizite Debatte darüber und keine wechselseitige, explizite Kritik. So können in der Plenumsdiskussion unwidersprochen Aussagen getätigt werden, wie die folgenden: „Wir haben ja alle schon mal gedacht, dass Flüchtlinge... Ich will jetzt nichts Falsches sagen.... na, ich sag's mal: Vormünder eigentlich bräuchten oder sowas, nicht nur für die Kids.... Sie brauchen Anstöße, Motivation; man müsste sie durch Mentoren belgeiten. Das wäre das, was man institutionell leisen kann; der Rest hängt vom Individuum ab." Dieser Debattenbeitrag steht neben der expliziten Aussage, dass die Zeit der Ideologie, dass man nur in Deutsch sprechen könne, vorbei sei (wobei „Deutsch“ häufig nicht genannt wird, sondern von Zielsprache und im Kontrast dazu von „Muttersprache“ gesprochen wird). Paternalistische („Das sind die Themen, die für unsere Migrant*innen anstehen“) und nationalisierend-kulturalisierende („Sie kommen aus Ländern, die nicht unsere westlichen Werte teilen“) finden sich gleichermaßen; es scheint so, als ob die fachliche Verständigung über DaF für Geflüchtete gänzlich ohne die Diskussion eines gesellschaftstheoretischen und -politischen Bezugsrahmens abläuft.

\section{Fazit und Ausblick}

Der Beitrag sollte zeigen, dass einige ehrenamtlich Lehrende die Probleme ihrer Schüler*innen über eine national-kulturell-sprachliche Zugehörigkeit erklären, während auch andere Erklärungs- bzw. Verstehensversuche zu beobachten sind. Die teilnehmende Beobachtung an einem DaF-Kongress hat darüber hinaus verdeutlicht, dass es im fachlichen Diskurs ein neues Paradigma gibt, welches durch die Einführung neuer Prämissen und Zielvorstellungen in der Lage ist, die Problemwahrnehmungen der ehrenamtlich Lehrenden zu transzendieren. Gleichzeitig werden Ansätze, die diesem neuen Paradigma folgen, ohne gesellschaftstheoretische und -politische Explikation verfolgt und bestehen so parallel zu widersprüchlichen Ansätzen, die die Einführung in „die korrekte Sprache“ (und darüber hinaus in eine „Leitkultur“) zum Ziel haben. Insofern scheinen die im Feld beobachteten divergenten Kategorien von Erklärungen möglicherweise weniger in einer mangelnden disziplinären Sozialisation begründet als in unterschiedlichen grundlegenden migrations- und diverstitätsbezogenen Einstellungen und Anschauungen, die in unterschiedlichen gesellschaftlichen Milieus beheimatet sind und die sich - auf anderem Niveau - auch in der Disziplin selbst wiederfinden.

Eine explizite Auseinandersetzung mit Konzepten der Migrationspädagogik (Dirim - Mecheril 2010) sowie dem Konzept der Postmigrantischen Gesellschaft (Foroutan 2019) würde es möglich machen, zu fragen, ob der DaF-Unterricht nicht Raum (und Sprache(n)) für heterogene Erinnerungs-, Erfahrungs- und Gegenwartsräume schaffen müsste. 


\section{Literaturverzeichnis}

Breidenstein, Georg (2006): Teilnahme am Unterricht. Ethnographische Studien zum Schülerjob. Wiesbaden: Springer VS.

Breidenstein, Georg (2010): Einen neuen Blick auf schulischen Unterricht entwickeln: Strategien der Befremdung. In: Heinzel, Friederike et al. (hrsg.): „Auf unsicherem Terrain“. Ethnographische Forschung im Kontext des Bildungs- und Sozialwesens. Wiesbaden: Springer VS, S. 205-215.

Dirim, İnci - Mecheril, Paul (2010): Die Sprache(n) der Migrationsgesellschaft. In: Mecheril, Paul et al. (hrsg.): Migrationspädagogik. Weinheim und Basel: Beltz, S. 99-120.

Foroutan, Naika (2019: Die postmigrantische Gesellschaft. Ein Versprechen der pluralen Demokratie. Bielefeld: transcript.

Hahn, Kathrin (2011): Alter, Migration und Soziale Arbeit. Zur Bedeutung von Ethnizität in Beratungsgesprächen der Altenhilfe. Bielefeld: transcript.

Knoblauchch, Hubert (2001): Fokussierte Ethnographie. Soziologie, Ethnologie und die neue Welle der Ethnographie. In: Sozialer Sinn 1/2001, S. 123-141.

Kühn, Thomas - Witzel, Andreas (2000): Der Gebrauch einer Textdatenbank im Auswertungsprozess problemzentrierter Interviews. In: Forum Qualitative Sozialforschung 1(3). http://www. qualitative-research.net/index.php/fqs/article/view/1035/2237 (20. 11. 2019).

Mayring, Philipp (2010a): Qualitative Inhaltsanalyse. Grundlagen und Techniken. 11., aktualisierte und überarb. Aufl. Weinheim: Beltz.

Mayring, Philipp (2010b): Qualitative Inhaltsanalyse. Forum Qualitative Sozialforschung, Volume 1, No 2. https://www.ph-freiburg.de/fileadmin/dateien/fakultaet3/sozialwissenschaft/Quasus/Volltexte/2-00mayring-d_qualitativeInhaltsanalyse.pdf (20. 11. 2019).

Rommelspacher, Birgit (2006): Dominanzkultur. Texte zu Fremdheit und Macht. Berlin: Orlanda Frauenverlag.

Schulz, Marc (2014): Ethnografische Beobachtung. In: Tillmann, Angela - Fleischer, Sandra Hugger, Kai-Uwe (hrsg.): Handbuch Kinder und Medien. Digitale Kultur und Kommunikation, Wiesbaden: Springer VS.

Strauss, Anselm - Corbin, Juliet (1990): Basics of Qualitative Research: Grounded Theory Procedures and Techniques. Thousand Oaks: SAGE Publications.

Wagner, Constantin (2016): Die Reproduktion „ethnisch“ vermittelter sozialer Ungleichheit in einem Schweizerischen Sozialamt. Folgen institutioneller und akteursbezogener Kategorisierungen. In: Arslan, Emre - Bozay, Kemal (hrsg.): Bildungsungleichheit und Symbolische Ordnung in der Migrationsgesellschaft, Wiesbaden: Springer VS, S. 419-437.

Wagner, Constantin (2017): Öffentliche Institutionen als weiße Räume? Rassismusreproduktion durch ethnisierende Kategorisierungen in einem schweizerischen Sozialamt. Bielefeld: transcript.

\footnotetext{
Jun.-Prof. Dr. Constantin Wagner / c.wagner@uni-mainz.de

Johannes Gutenberg Universität Mainz, FB 02: Sozialwissenschaften, Medien und Sport, Institut für Erziehungswissenschaft Jakob-Welder-Weg 12, 55128 Mainz, DE
}

This work can be used in accordance with the Creative Commons BY-SA 4.0 International license terms and conditions (https://creativecommons.org/licenses/by-sa/4.0/legalcode). This does not apply to works or elements (such as image or photographs) that are used in the work under a contractual license or exception or limitation to relevant rights 